\title{
Chronic endurance exercise induces quadriceps nitrosative stress in patients with severe COPD
}

\author{
E Barreiro, ${ }^{1}$ R Rabinovich, ${ }^{2}$ J Marin-Corral, ${ }^{1}$ J A Barberà, ${ }^{2}$ J Gea, ${ }^{1}$ J Roca ${ }^{2}$
}

- Supplementary data are published online only at http:// thorax.bmj.com/content/vol64/ issue1

${ }^{1}$ Muscle and Respiratory System Research Unit and Respiratory Medicine Department, IMIM-Hospital del Mar, Barcelona, Centro de Investigación en Red de Enfermedades Respiratorias (CibeRes), Health and Experimental Sciences Department (CEXS), Universitat Pompeu Fabra (UPF), and Medical School, Universitat Autònoma de Barcelona (UAB), Barcelona, Catalonia, Spain; ${ }^{2}$ Servei de Pneumologia (ICT), Hospital Clinic, IDIBAPS, Centro de Investigación en Red de Enfermedades Respiratorias (CibeRes), Universitat de Barcelona, Barcelona, Catalonia, Spain

Correspondence to: Dr E Barreiro, Muscle and Respiratory System Research Unit, IMIM, C/Dr Aiguader, 88, Barcelona, E-08003 Spain; ebarreiro@imim.es

Received 22 July 2008 Accepted 29 July 2008

Published Online First

3 October 2008

\begin{abstract}
Background: Although exercise training has beneficial effects on skeletal muscle bioenergetics and exercise performance in patients with severe chronic obstructive pulmonary disease (COPD), it may also be associated with increased quadriceps oxidative and nitrosative stress. The aim of this study was to explore quadriceps oxidative and nitrosative stress in patients with severe COPD, both before and after a 3 week endurance exercise programme, and to identify the nature of the oxidatively modified proteins.
\end{abstract}

Methods: Reactive carbonyls, hydroxynonenal-protein adducts, antioxidant enzymes, nitric oxide synthase (NOS) and 3-nitrotyrosine levels were determined in the quadriceps (pre- and post-exercise) of 15 patients with severe COPD and seven healthy controls using immunoblotting (one- and two-dimensional electrophoresis), activity assays and mass spectrometry.

Results: At baseline, muscle levels of reactive carbonyls, which were negatively associated with muscle strength and exercise tolerance, were significantly higher in patients than in controls. Moreover, baseline hydroxynonenal-protein adducts, superoxide dismutase activity, inducible NOS and 3-nitrotyrosine immunoreactivity levels were also significantly increased in the quadriceps of patients compared with controls. In patients, chronic exercise induced a significant rise in inducible NOS levels and a fourfold increase in protein nitration. Chronic endurance exercise induced tyrosine nitration of muscle enolase $3 \beta$, aldolase $A$, triosephosphate isomerase, creatine kinase, carbonic anhydrase III, myoglobin and uracil DNA glycosylase in the quadriceps of patients, while contractile protein alpha-1 actin was nitrated only in patients exhibiting muscle loss (post hoc analysis).

Superoxide dismutase activity increased after the exercise programme only in controls.

Conclusions: In severe COPD, chronic endurance exercise induces increased tyrosine nitration of quadriceps proteins involved in glycolysis, energy distribution, carbon dioxide hydration, muscle oxygen transfer, DNA repair and contractile function in patients exhibiting systemic effects of the disease.

Poor exercise tolerance and impaired quality of life partially owing to peripheral muscle dysfunction are major complaints among patients with chronic obstructive pulmonary disease (COPD). It is well established that endurance training has beneficial effects on skeletal muscle bioenergetics and exercise performance in these patients, ${ }^{1-4}$ and is an essential component in pulmonary rehabilitation programmes. ${ }^{56}$ Several lines of evidence indicate that high intensity training, targeted above 60 $70 \%$ peak work rate, is required to improve skeletal muscle oxygen transport, decrease blood lactate levels and enhance mitochondrial oxidative capacity in patients with moderate to severe COPD. ${ }^{13} 478$

One could argue, however, that in patients with COPD, chronic endurance exercise of high intensity might be associated with increased production of reactive oxygen and reactive nitrogen species (RNS) in the quadriceps, exceeding antioxidant capacity in the tissue, thus leading to muscle oxidative stress. Indeed, oxidative stress has been considered to be a major player in the peripheral muscle dysfunction of patients with COPD, both at rest and after exercise. ${ }^{9-14}$ In line with this, we have previously demonstrated that dogs subjected to high intensity inspiratory loads for 2 weeks showed a significant increase in oxidant induced protein modifications in their diaphragms, which was neutralised by concomitant treatment of the animals with the antioxidant $\mathrm{N}$-acetyl-cysteine. ${ }^{15}$ In patients with COPD, antioxidant therapy ${ }^{12}$ also attenuated exercise induced muscle oxidative stress, partially restoring muscle function. Furthermore, other reports also demonstrated ${ }^{14}{ }^{16}$ a decrease in muscle redox potential after endurance training in patients with severe COPD, as opposed to healthy sedentary subjects in whom training markedly enhanced muscle glutathione levels.

On the other hand, experimental studies showed that alterations in the nitroso-redox balance of cardiomyocytes were associated with abnormally high energy requirements at a given work rate. ${ }^{17}$ Interestingly, patients with COPD also exhibited lower limb mechanical inefficiency ${ }^{48}$ as well as abnormal high energy expenditure. ${ }^{19}$

Hence, taking all of these data together, it is reasonable to anticipate that chronic endurance exercise of relatively high intensity will enhance oxidative and nitrosative stress in the quadriceps of patients with severe COPD, possibly worsening their muscle dysfunction. ${ }^{20}$ Enhanced muscle oxidative stress could, therefore, counteract the beneficial effects of endurance exercise in specific subsets of patients with COPD in whom complementary pharmacological interventions might be needed. Accordingly, our objectives were to explore, before and after a 3 week endurance exercise programme, quadriceps oxidative and nitrosative stress in patients with severe COPD, covering a wide spectrum of body mass composition, and in healthy controls. Moreover, the oxidatively modified proteins were also identified both before and after the endurance exercise programme in patients and controls.

\section{METHODS}

See additional information in the online supplement. 


\section{Subjects}

This was a hospital based study in which a group of 15 Caucasian male patients with stable severe $\mathrm{COPD}^{5}$ and a group of seven healthy male age matched controls were recruited on an outpatient basis at Hospital Clinic, Barcelona, Spain. Both patients and controls were studied in 2004-2005. Some of the data shown in table 1 were previously reported in another study exploring myofibre respiratory function. ${ }^{21}$ All patients with COPD and controls were studied at baseline, but two patients with COPD and two healthy subjects refused to undergo the second evaluation after the 3 week exercise programme and were therefore excluded from the analysis at that time point.

The current investigation was designed in accordance with both the ethical standards on human experimentation in our institution and the World Medical Association guidelines for research on human beings. The Ethics Committee on Human Investigation at Hospital Clinic approved all of the experiments. Informed written consent was obtained from all individuals.

\section{Chronic endurance exercise programme}

Patients and controls underwent a 3 week endurance exercise programme on a cycloergometer 5 days a week for three consecutive weeks. One $1 \mathrm{~h}$ session/day was established in all subjects, with effective exercise periods of at least $30 \mathrm{~min}$ in each case.

\section{Muscle biopsies}

Open muscle biopsies of the vastus lateralis were obtained before (at baseline) and after the 3 week endurance exercise programme, following the procedures published elsewhere. ${ }^{9} 10$

\section{Muscle biology analyses}

All muscle biology analyses were conducted blind in the same laboratory, at IMIM-Hospital del Mar-Universitat Pompeu Fabra.

Immunoblotting: one-dimensional electrophoresis

The effects of oxidants on muscle proteins were evaluated according to methodologies published elsewhere. ${ }^{9-11} 15$

Identification of tyrosine nitrated muscle proteins: two-dimensional electrophoresis

Tyrosine nitrated proteins were identified in the vastus lateralis before and after the 3 week endurance exercise programme following methodologies previously published. ${ }^{10} 22$

Identification of tyrosine nitrated muscle proteins: mass spectrometry

Identification of nitrated proteins was conducted in the Proteomics Laboratory at Universitat Pompeu Fabra, as published elsewhere. ${ }^{102}$

\section{Superoxide dismutase and catalase activity assays}

Commercially available superoxide dismutase (SOD) and catalase assay kits (Cayman Chemical Co, Ann Arbor, Michigan, USA) were used according to the corresponding manufacturer's instructions.

\section{Statistical analysis}

Results are presented as mean (SD). Comparisons of baseline physiological and biological variables between healthy sedentary subjects and patients with COPD were assessed using the unpaired Student's t test. Chronic exercise induced changes in muscle biological variables were explored using the paired Student's $t$ test. Relationships between physiological and biological variables were assessed using Pearson's correlation coefficient.

We conducted a post hoc analysis in which physiological and biological variables were compared in patients with a low fat free mass index (FFMI) $\left(F_{F M I} \leqslant 18 \mathrm{~kg} / \mathrm{m}^{2}\right),{ }^{23}$ seven of 15 patients, and in patients with a normal FFMI $\left(\mathrm{FFMI}_{\mathrm{N}}\right)$, using one way ANOVA together with Tukey's test to adjust for multiple comparisons.

Sample size was calculated on the basis of assumptions of $80 \%$ power to detect an improvement of $20 \%$ or more in measured outcomes at a level of significance of $p \leqslant 0.05$ as well as on previously published studies using similar physiological and biological approaches. ${ }^{9-16} 20$

\section{RESULTS}

\section{Characteristics of the study groups and physiological effects of training}

See additional information in the online supplement.

No significant differences were found in age, body mass index (BMI) and FFMI between controls and patients with COPD (table 1). As expected, lung function impairment indicating severe disease was observed in patients. Moreover, patients with COPD showed reduced isometric and isokinetic quadriceps strength and lower exercise tolerance compared with controls.

\section{Muscle oxidative stress}

See additional information in the online supplement.

\section{Muscle redox balance}

At baseline, total protein carbonylation levels were significantly higher in the quadriceps of patients with COPD compared with controls (fig 1A). Among all patients with COPD, significant

Table 1 Anthropometric characteristics and functional status of the study groups

\begin{tabular}{|c|c|c|}
\hline & $\begin{array}{l}\text { Control subjects } \\
(\mathrm{n}=7)\end{array}$ & $\begin{array}{l}\text { COPD patients } \\
(n=15)\end{array}$ \\
\hline Age (years) & $62(6)$ & $62(7)$ \\
\hline BMI $\left(\mathrm{kg} / \mathrm{m}^{2}\right)$ & $27.9(5.1)$ & $24.4(6.2)$ \\
\hline FFMI $\left(\mathrm{kg} / \mathrm{m}^{2}\right)$ & $22.3(2.5)$ & $20.0(3.5)$ \\
\hline $\mathrm{FEV}_{1}$ (I) & $3.2(0.5)$ & $1.4(0.6)^{* * *}$ \\
\hline $\mathrm{FEV}_{1}(\%$ pred) & $95(15)$ & $41(18)^{* * *}$ \\
\hline FVC (\% pred) & $97(12)$ & $68(18)^{* * *}$ \\
\hline $\mathrm{FEV}_{1} / \mathrm{FVC}(\%)$ & $78(5)$ & $44(12)^{* * *}$ \\
\hline RV (\% pred) & NA & $177(48)$ \\
\hline Tlco (\% pred) & NA & $50(21)$ \\
\hline $\mathrm{PaO}_{2}(\mathrm{kPa})$ & $12.6(1.7)$ & $9.9(1.3)^{*}$ \\
\hline $\mathrm{PaCO}_{2}(\mathrm{kPa})$ & $4.9(0.5)$ & $5.6(0.8)$ \\
\hline 0 isometric $(\mathrm{Nm})$ & $205(31)$ & $153(58)^{*}$ \\
\hline 0 isokinetic $(\mathrm{Nm})$ & $129(30)$ & $92(43)^{*}$ \\
\hline 0 endurance $(\mathrm{J})$ & 2200 (599) & $1480(725)^{* *}$ \\
\hline $\mathrm{V}_{2}$ peak $(\%$ pred) & $100(16)$ & $57(30)^{*}$ \\
\hline WRpeak (\% pred) & $100(18)$ & $48(25)^{*}$ \\
\hline
\end{tabular}

Data are presented as mean (SD).

BMI, body mass index; COPD, chronic obstructive pulmonary disease; $\mathrm{FEV}_{1}$, forced expiratory volume in $1 \mathrm{~s} ; \mathrm{FEV}_{1} / \mathrm{FVC}, \mathrm{FEV}_{1} / \mathrm{FVC}$ ratio expressed as actual percentage; FFMI, fat free mass index; FVC, forced vital capacity; J, Jules; NA, not available; Nm, Newton metres; $\mathrm{PaCO}_{2}$, arterial carbon dioxide tension; $\mathrm{PaO}_{2}$, arterial oxygen tension; pred, predicted; $\mathrm{Q}$, quadriceps force; RV, residual volume; TIco, carbon monoxide transfer factor; $\mathrm{V}_{2}$ peak, peak exercise oxygen uptake; WRpeak, peak work rate. ${ }^{*} p \leqslant 0.05,{ }^{* *} p=0.06,{ }^{* *} p \leqslant 0.001$ between patients with COPD and control subjects. 
Figure 1 (A) Individual and mean group effects of a 3 week endurance exercise programme on protein carbonylation in the quadriceps of control subjects $(n=7)$ and in patients with chronic obstructive pulmonary disease (COPD) ( $n=15)$. At baseline, mean values of total reactive carbonyls were higher in patients than in controls. The 3 week endurance exercise programme did not significantly modify total muscle protein carbonylation in any of the study groups. In the post hoc analysis, at baseline, mean values of protein carbonylation were significantly greater in the muscles of patients with a low fat free mass index $\left(\mathrm{FFMI}_{\mathrm{L}}\right)$ than in either those with a normal fat free mass index $\left(F F M I_{N}\right)$ or in controls. Chronic endurance exercise did not significant change protein carbonylation levels, compared with baseline, in any of the subgroups of patients with COPD. Note that two patients with COPD (one $\mathrm{FFMI}_{\mathrm{L}}$ and one $\mathrm{FFMI}_{\mathrm{N}}$ ) and two healthy subjects refused to undergo the second evaluation after the 3 week exercise programme.

(B) At baseline, among all patients with COPD, muscle protein carbonylation inversely correlated with quadriceps isometric force and oxygen uptake $\left(\mathrm{FFMI}_{\mathrm{L}}\right.$ and $\mathrm{FFMI}_{\mathrm{N}}$ patients are represented by the filled and open symbols, respectively). GADPH, glyceraldehyde-3-phosphate dehydrogenase; Nm, Newton metres; 0 , quadriceps force.
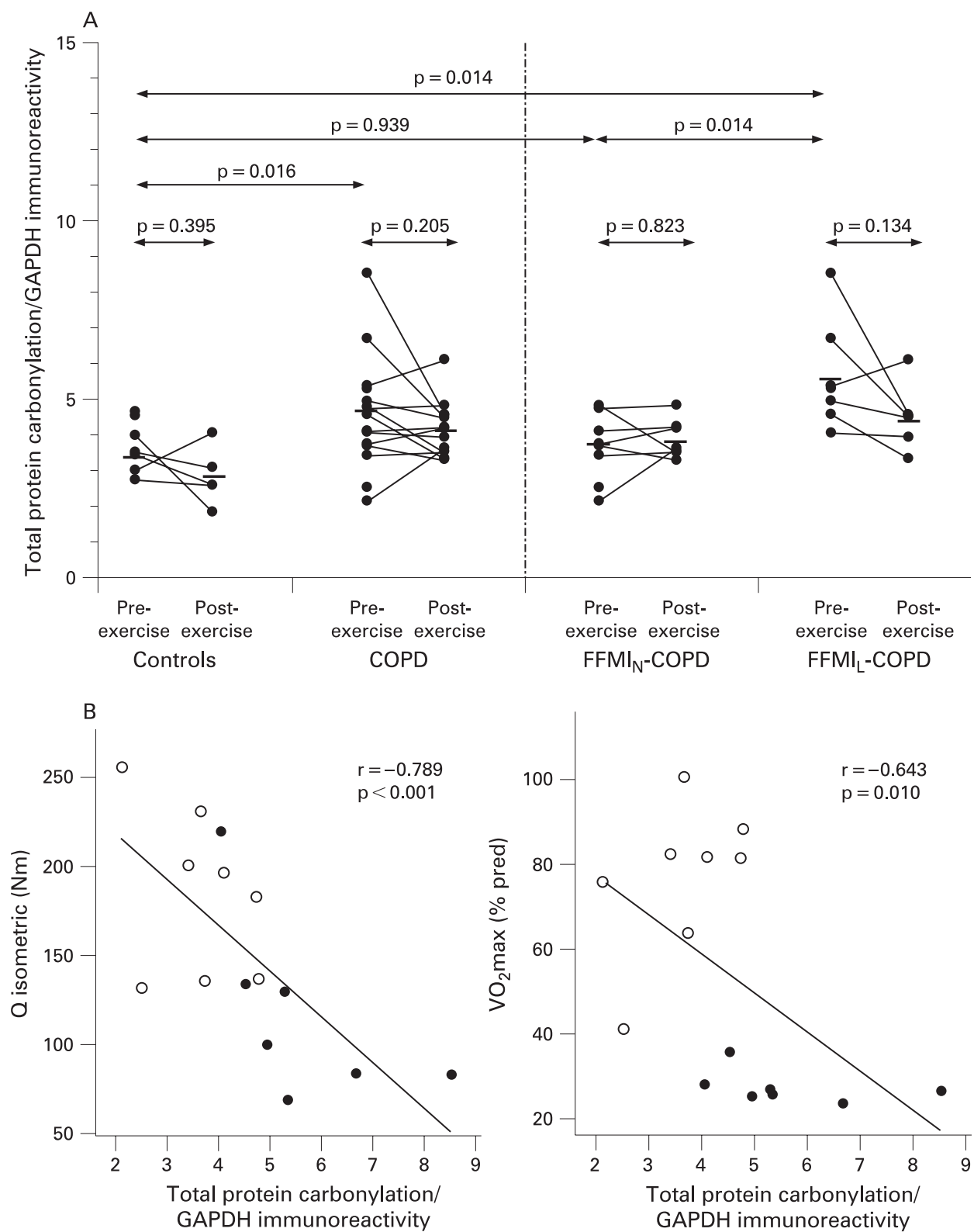

negative correlations were found between baseline muscle reactive carbonyl groups and FFMI $(r=-0.621, p=0.013)$, quadriceps strength and exercise tolerance (fig 1B). After the 3 week endurance exercise programme, muscle reactive carbonyl groups did not significantly differ from baseline values in either controls or patients with COPD (fig 1A). At baseline, hydroxynonenal (HNE)-protein adducts were significantly higher in patients with COPD than in controls (fig 2). Among all patients with COPD, no significant relationships were found between baseline HNE-protein adduct levels and physiological variables. Chronic endurance exercise induced a significant rise in HNEprotein adducts in both healthy controls and patients with COPD (fig 2). Baseline muscle SOD activity levels were significantly greater in patients with COPD than in controls (see fig $3 \mathrm{C}$ in the online supplement). However, chronic endurance exercise induced a significant increase in muscle SOD activity only in controls (see fig $3 \mathrm{C}$ in the online supplement).

At baseline, $\mathrm{FFMI}_{\mathrm{L}}$ patients exhibited higher levels of muscle reactive carbonyl groups than either healthy subjects or $\mathrm{FFMI}_{N}$ patients (fig 1A). The 3 week endurance exercise programme did not induce any significant change in total reactive carbonyls in either subgroup of patients with COPD. Compared with controls, baseline HNE-protein adducts were significantly greater in the quadriceps of both subgroups of patients, and were further increased by chronic endurance exercise (fig 2).

\section{Muscle nitrosative stress}

See additional information in the online supplement.

Protein levels of nitric oxide synthase

At baseline, muscle inducible nitric oxide synthase (iNOS) protein levels were greater in patients with COPD compared with controls (see fig 4D in the online supplement) Interestingly, chronic endurance exercise induced a significant increase in iNOS protein content in the quadriceps of patients with COPD but not in healthy controls (see fig 4D in the online supplement). 
Figure 2 Individual and mean group effects of a 3 week endurance exercise programme on hydroxynonenal (HNE)protein adducts in the quadriceps of control subjects $(n=7)$ and patients with chronic obstructive pulmonary disease (COPD) ( $n=15)$. At baseline, mean values of HNE-protein adducts were higher in patients than in controls. The 3 week endurance exercise programme significantly increased total muscle HNEprotein adducts in both control subjects and patients with COPD. In the post hoc analysis, at baseline, mean values of HNE-protein adducts were significantly greater in the muscles of patients with a normal fat free mass index $\left(\mathrm{FFMI}_{\mathrm{N}}\right)$ and a low fat free mass index $\left(F F M I_{L}\right)$ than in controls. Total muscle HNE-protein adducts levels significantly increased in both subgroups of patients after chronic exercise compared with baseline. Note that two patients with COPD (one FFMI and one $\mathrm{FFMI}_{\mathrm{N}}$ ) and two healthy subjects refused to undergo the second evaluation after the 3 week exercise programme. GADPH, glyceraldehyde-3-phosphate dehydrogenase.

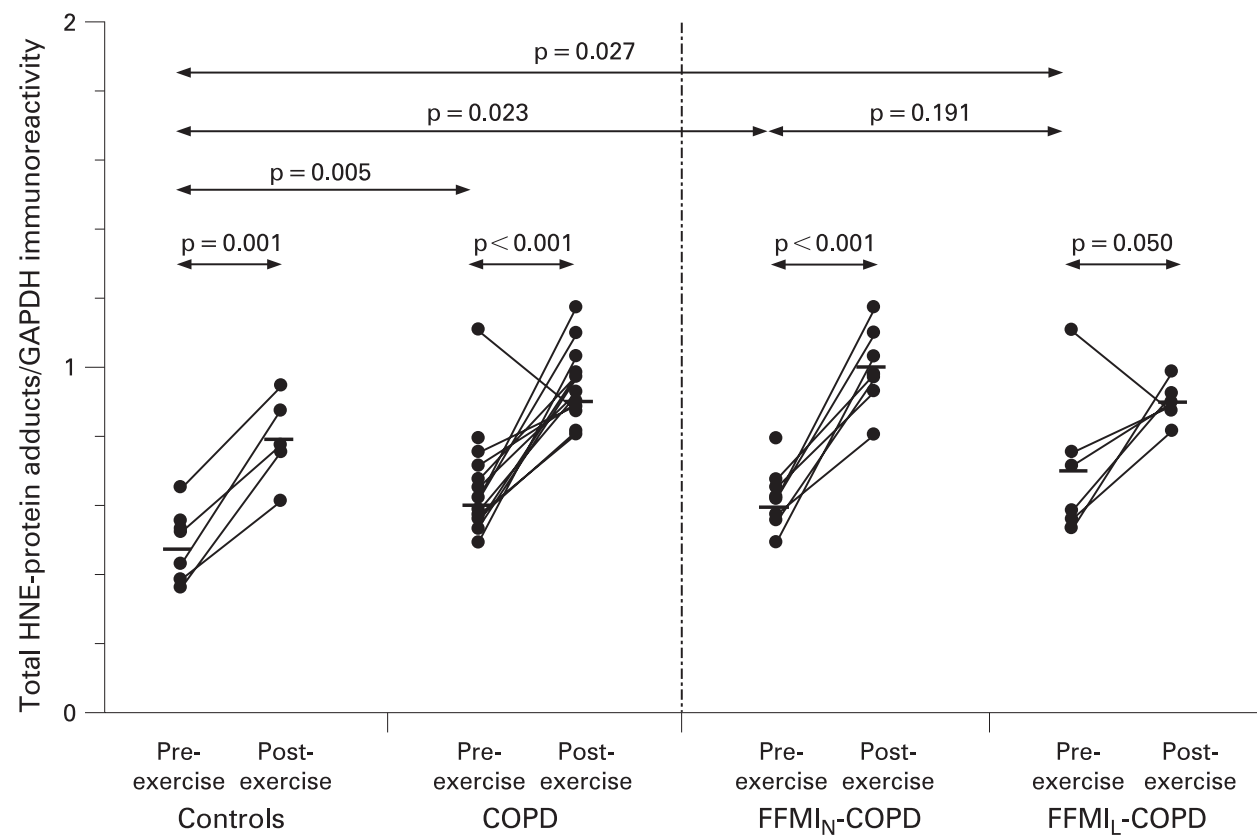

Protein tyrosine nitration

At baseline, protein tyrosine nitration levels were significantly higher in patients with COPD than in controls (fig $3 \mathrm{~A}$ ). Chronic endurance exercise induced a significant but dissimilar rise in protein tyrosine nitration in patients with COPD (66 (26)\%) compared with controls (15 (9) \%; p<0.001) (fig 3A). Furthermore, after the 3 week exercise programme, a negative correlation was observed between muscle protein nitration levels and peak oxygen uptake, which was not seen at baseline (fig 3B). Identification of the different tyrosine nitrated proteins in two control subjects and five patients with severe COPD, using two-dimensional electrophoresis and mass spectrometry, revealed that glycolytic enzymes (muscle specific enolase $3 \beta$, aldolase A and triosephosphate isomerase), muscle creatine kinase, carbonic anhydrase III, uracil DNA glycosylase and myoglobin were specifically nitrated in the vastus lateralis of patients with COPD and controls before and after chronic exercise (table 2).

Baseline iNOS levels were greater in the quadriceps of both $F_{N} M_{N}$ and $F F M I_{L}$ patients than in control subjects, while chronic exercise induced a significant increase in muscle iNOS only in FFMI $I_{L}$ patients (see fig 4D in the online supplement). At baseline, both FFMI $\mathrm{N}_{\mathrm{N}}$ and FFMI $\mathrm{L}$ patients exhibited significantly greater levels of muscle protein tyrosine nitration than healthy controls (fig 3A). Chronic endurance exercise induced increase in muscle protein nitration was higher in $\mathrm{FFMI}_{\mathrm{L}}$ patients than in those with $\mathrm{FFMI}_{\mathrm{N}}(85(24) \%$ and 50 (15)\%, respectively; $\mathrm{p}<0.05$ ) (fig $3 \mathrm{~A}$ ). Interestingly, the contractile actin was identified to be tyrosine nitrated only in $\mathrm{FFMI}_{\mathrm{L}}$ patients after chronic exercise (table 2).

\section{DISCUSSION}

The main findings in this study were that in the vastus lateralis from clinically stable patients with severe COPD compared with controls: (1) at baseline, protein carbonylation levels were significantly greater and inversely correlated with quadriceps strength, exercise capacity and FFMI; (2) baseline levels of SOD activity, HNE-protein adducts, iNOS protein and protein tyrosine nitration were significantly increased; (3) a 3 week exercise programme induced a significant but similar rise in muscle HNE-protein adducts in both patients and controls, while it was only in patients that it induced a rise in iNOS levels endurance exercise induced tyrosine nitration of glycolytic enzymes, creatine kinase, carbonic anhydrase III, myoglobin and uracil DNA glycosylase in all the patients with COPD, while alpha-1 actin was tyrosine nitrated only in $\mathrm{FFMI}_{\mathrm{L}}$; (5) muscle SOD activity increased after the exercise programme change in either patients or controls.

\section{Muscle oxidative stress}

Peripheral muscle dysfunction has long been recognised as a major problem in patients with COPD, contributing to poor exercise tolerance and reduced quality of life. ${ }^{24}{ }^{25}$ The current findings confirm results from previous studies where oxidative stress was shown to be involved in the peripheral muscle dysfunction of patients with COPD both at rest and after exercise. $^{9-14} 1620$ However, what is novel about the findings in the present investigation is that in all patients with COPD, muscle protein carbonylation was inversely related to quadriceps force, independent of FFM, to exercise capacity and to FFMI. Interestingly, in a recent study conducted by some of the authors, ${ }^{20}$ muscle protein carbonylation levels were also shown to negatively correlate with quadriceps force among a population of stable patients with severe COPD. Collectively, these findings suggest that increased muscle protein oxidation is probably a major contributor to the peripheral muscle dysfunction of patients with severe COPD.

In the present study, chronic endurance exercise induced a significant increase in HNE-protein adducts in the quadriceps of both patients and healthy controls. It can be concluded from these observations that oxidants physiologically generated by chronic exercise have also targeted membrane lipids within the and a fourfold increase in protein nitration; (4) chronic only in controls while catalase content or activity did not 
Figure 3 (A) Individual and mean group effects of a 3 week endurance exercise programme on protein tyrosine nitration in the quadriceps of control subjects $(n=7)$ and patients with chronic obstructive pulmonary disease (COPD) $(n=15)$. At baseline, mean values (SD) of protein than in controls. Chronic endurance exercise induced a fourfold increase in protein tyrosine nitration in patients with COPD. In the post hoc analysis, at baseline, mean values of 3-nitrotyrosine immunoreactivity were significantly greater in the muscles of patients with a normal fat free mass index $\left(\mathrm{FFMI}_{\mathrm{N}}\right)$ and a low fat free mass index (FFMI $\mathrm{L}_{\mathrm{L}}$ compared with controls. Moreover, the chronic exercise induced in protein nitration was significantly greater in $\mathrm{FFMI}_{\mathrm{L}}$ than in $\mathrm{FFMI}_{\mathrm{N}}$ patients. Note that two patients with COPD (one FFMI $\mathrm{L}_{\mathrm{L}}$ and one $\mathrm{FFMI}_{N}$ ) and two healthy subjects refused to undergo the second evaluation after the 3 week exercise programme. (B) Among all patients with COPD, after the 3 week endurance exercise programme, quadriceps protein nitration inversely correlated with exercise capacity, as measured by peak oxygen uptake (righthand side panel) (FFMI and $F F M I_{N}$ patients are represented in filled and open symbols, respectively). Note that only 13 patients with COPD are depicted in the graph as two patients refused to undergo the second evaluation. At baseline, however, quadriceps protein nitration did not significantly correlate with exercise capacity ( $p>0.05$, left-hand side panel). GADPH, glyceraldehyde-3-phosphate dehydrogenase; pred, predicted; $\mathrm{V}_{2} \mathrm{max}$, peak exercise oxygen uptake. tyrosine nitration were higher in patients
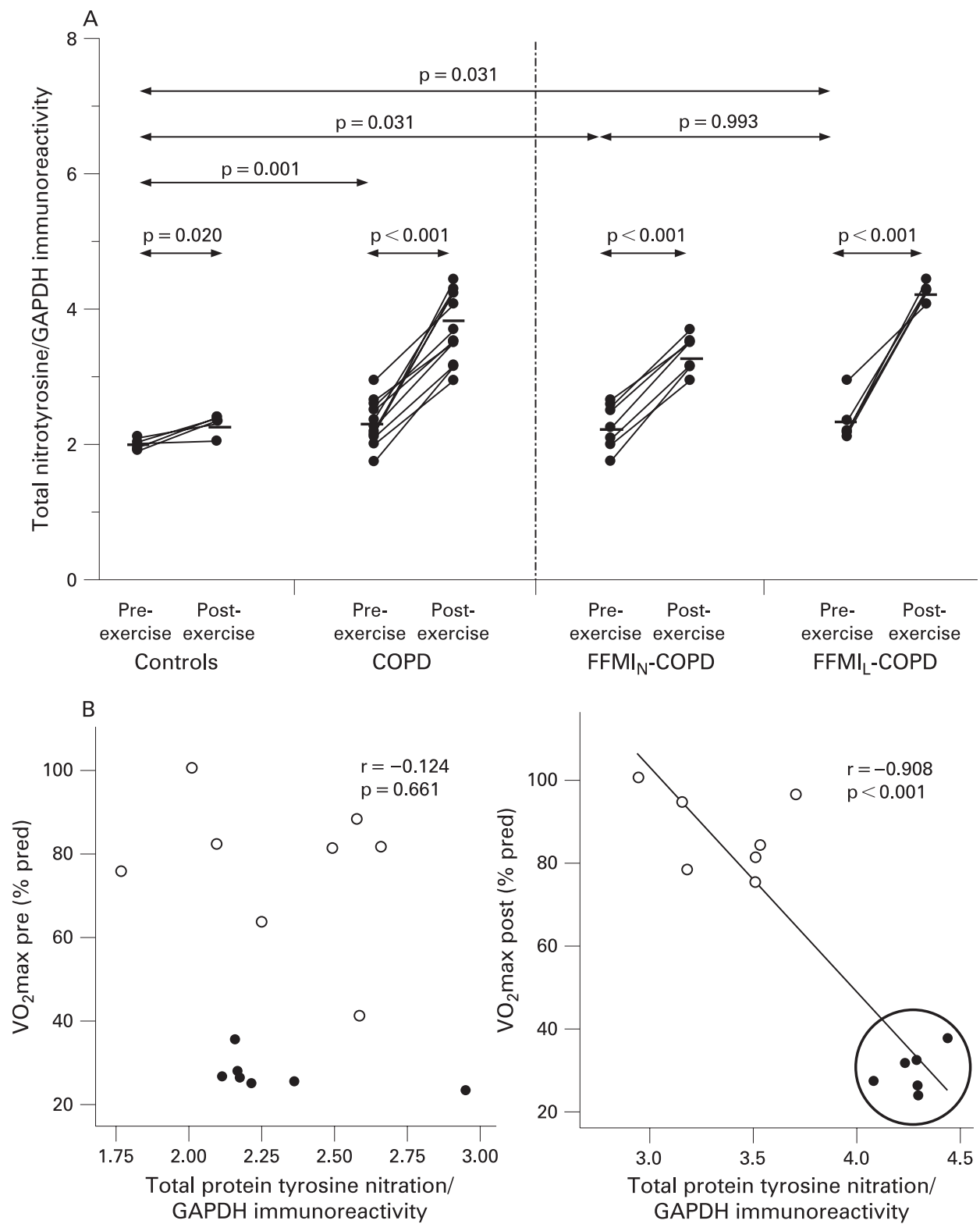

myofibres, thus inducing post-translational oxidative modifications of muscle proteins in both patients with COPD and controls. Furthermore, in this study, antioxidant mechanisms (SOD activity) were increased after chronic exercise only in the limb muscles of healthy controls but not in those of patients.
This is in keeping with previous studies conducted by our group ${ }^{14}{ }^{16}$ in which antioxidant mechanisms were shown to be significantly induced after exercise training in the muscles of control subjects but not in patients with severe COPD. One likely explanation for the lack of induction of SOD activity in

Table 2 Identified proteins in the muscle lysates of the vastus lateralis of patients with COPD and in control subjects

\begin{tabular}{|c|c|c|c|c|c|}
\hline Study subjects & Identified nitrated proteins & $\begin{array}{l}\text { Accession } \\
\text { No }\end{array}$ & Mass & $\begin{array}{l}\text { MASCOT } \\
\text { score }\end{array}$ & $\begin{array}{l}\text { Peptide } \\
\text { matched }\end{array}$ \\
\hline Ctl and COPD pre and post & Muscle specific enolase $3 \beta$ & $\mathrm{gi} / 16878083$ & 47244 & 129 & 7 \\
\hline Ctl and COPD pre and post & Aldolase A & gi/229674 & 39720 & 116 & 4 \\
\hline Ctl and COPD pre and post & Chain $A$, triosephosphate isomerase & gi/999892 & 26807 & 145 & 7 \\
\hline Ctl and COPD pre and post & Muscle creatine kinase & gi/119577741 & 45971 & 187 & 16 \\
\hline Ctl and COPD pre and post & Carbonic anhydrase III & $\mathrm{gi} / 224979$ & 29707 & 145 & 8 \\
\hline Ctl and COPD pre and post & Myoglobin & $\mathrm{gi} / 229361$ & 17099 & 101 & 14 \\
\hline Ctl and COPD pre and post & Uracil DNA glycosylase & $\mathrm{gi} / 35053$ & 35698 & 99 & 7 \\
\hline Only FFMIL COPD post & Alpha-1 actin precursor & $\mathrm{gi} / 4501881$ & 42366 & 107 & 9 \\
\hline
\end{tabular}

$\mathrm{Ctl}$, control; COPD, chronic obstructive pulmonary disease; $\mathrm{FFMI}_{\mathrm{L}}$, patients with a low fat free mass index; post, after the 3 week endurance exercise programme; pre, before the 3 week endurance exercise programme. 
the muscles of our patients after chronic exercise is that the baseline levels of this enzyme were much greater in patients than in controls. Clearly, further studies will be necessary in order to explore the potential induction of antioxidant mechanisms in the peripheral muscles of patients with severe COPD after chronic exercise programmes.

\section{Muscle nitrosative stress}

The present study is the first to report that chronic exercise induced a substantial increase in quadriceps nitrosative stress in patients with severe COPD. This phenomenon was accompanied by the inefficiency of the muscle antioxidant systems to adapt to the changes generated by chronic exercise in these patients.

Protein tyrosine nitration is considered to be a marker of excessive RNS production in tissues. Peroxynitrite, which is formed from the near diffusion limited reaction between nitric oxide and superoxide anions, accounts for most of protein tyrosine nitration in skeletal muscles. ${ }^{26}$ In the present study, muscle levels of the constitutive nNOS and eNOS did not differ between patients and controls at baseline. However, baseline levels of iNOS, although weak, were detected in both patients with COPD and controls and were significantly greater in the quadriceps of the patients. These findings are in agreement with two previous studies ${ }^{27}{ }^{28}$ in which iNOS protein levels were shown to be increased in the quadriceps of patients with COPD and weight loss. However, they certainly contrast with a previous study conducted by our group, ${ }^{9}$ in which iNOS could not be detected in the vastus lateralis of patients with moderate COPD and normal weight. In light of all of these studies, we can conclude that increasing severity of COPD and weight loss probably influence iNOS content in the peripheral muscles of these patients.

The increase in protein tyrosine nitration observed in patients with severe COPD and control subjects after chronic exercise was probably attributable to the action of different NOS in each group: whereas constitutive eNOS was probably the main contributor in the $\mathrm{FFMI}_{\mathrm{N}}$ and control subjects, iNOS was a major contributor to nitric synthesis in the FFMI $\mathrm{L}_{\mathrm{L}}$ patients. This clearly contrasts with a previous study ${ }^{29}$ in which rats exposed to a 4 week treadmill training showed increases in protein nitration and in constitutive NOS isoforms, but not in iNOS, in their gastrocnemius. Differences in the two study models and species probably account for such discrepancies.

In order to understand the pathophysiological consequences of protein nitration, the modified proteins were identified in the current investigation. To the best of our knowledge, this study is the first to report that proteins involved in glycolysis, energy distribution, carbon dioxide hydration, oxygen transport, DNA repair and contractile function were shown to be posttranslationally modified by RNS in the quadriceps of patients with severe COPD at rest and after chronic exercise. Protein tyrosine nitration, a very selective phenomenon, ${ }^{30}$ is associated with several important diseases, including neurological disorders, ${ }^{31}$ cardiovascular pathology, ${ }^{32}$ acute respiratory distress syndrome ${ }^{33}$ and transplant rejection. ${ }^{34}$ Protein nitration may result in either gain (fibrinogen) ${ }^{33}$ or loss of protein function, ${ }^{33-35}$ and in increased protein degradation by the proteasome. ${ }^{36}$ Nitration of tyrosine residues in these proteins may not be the only post translational modification responsible for alterations in protein function as oxidation of other critical amino acids may also occur in proteins sensitive to both oxidation and nitration. In fact, recent observations ${ }^{22}{ }^{37}$ have also demonstrated that muscle specific enolase, aldolase, triosephosphate isomerase-1, creatine kinase, carbonic anhydrase III and alphaactin were oxidised in the diaphragms of endotoxaemic rats, while creatine kinase and carbonic anhydrase III were carbonylated in the quadriceps of patients with moderate COPD at rest. ${ }^{10}$

Tyrosine nitration of the proteins identified in the current investigation has already been reported in previous studies using in vivo and in vitro models. For instance, the enzymes $\beta$-enolase, triosephosphate isomerase and carbonic anhydrase III were tyrosine nitrated with increasing age in old rats. ${ }^{38}$ In another study, peroxynitrite inhibited the activity of fructose biphosphate aldolases inducing a concomitant increase in nitrotyrosine immunoreactivity. ${ }^{39}$ Myofibrillar creatine kinase was also a sensitive target for peroxynitrite mediated nitration with concomitant enzyme inhibition in the failing heart, ${ }^{40}$ suggesting a pivotal role for this phenomenon in the alterations of cardiomyocyte energetics. Interestingly, the function of human myoglobin was also impaired after modifications induced by RNS. ${ }^{41}$ More recently, exposure to peroxynitrite of cardiac myofibrillar proteins, including actin, was shown to inhibit protein function in an in vitro motility assay. ${ }^{42}$ Lately, uracil DNA glycosylase nitration has also been proposed as an indicator of DNA nitrosative damage. ${ }^{43}$ On the basis of these findings, we can predict that peroxynitrite mediated nitration of the proteins identified in our study will contribute, at least in part, to muscle contractile dysfunction, thus probably playing a role in the quadriceps mechanical inefficiency exhibited by patients with severe COPD. ${ }^{4}{ }^{18}{ }^{19}$ Clearly, a further study would be required in order to specifically explore the functional alterations of the nitrated proteins in the quadriceps of these patients both at rest and after chronic exercise.

\section{Study limitations}

One limitation in the current investigation has to do with the relatively small number of subjects studied. On the basis of the relatively "invasive" nature of this investigation with both patients with severe COPD and healthy controls undergoing two open muscle biopsies, two maximal exercise tests and a 3 week programme of high intensity exercise, and on the fact that two patients with COPD and two healthy controls refused to undergo the second evaluation, we felt discouraged to recruit more patients and controls for the purpose of this investigation. Moreover, significant results were found with this relatively small population. Therefore, from an ethical point of view, utilisation of a larger population size than that required for obtaining significant results is not advisable.

Another limitation was that patients exhibiting lower FFMI were also those showing worse lung function. Indeed, it is well known that weight loss and increasing severity of COPD are not entirely separate phenomena and that their associations are linked to poor prognosis of the disease. Although our population of patients with COPD was rather small to determine the exact contribution of either impaired lung function or muscle loss to the study results, increasing disease severity and nutritional depletion have already been shown to be associated in previous studies $^{44}{ }^{45}$ (see additional information in the online supplement).

\section{CONCLUSIONS}

This study is the first to report that in severe COPD, chronic endurance exercise induces increased tyrosine nitration of quadriceps proteins involved in glycolysis, energy distribution, carbon dioxide hydration, muscle oxygen transfer, DNA repair 
and contractile function in patients exhibiting systemic effects of the disease. This suggests that chronic endurance exercise of relatively high intensity in these patients may generate further disruption of the nitroso-redox balance leading to maladaptive muscle alterations that are not counteracted by the antioxidant mechanisms.

Acknowledgements: The authors thank Dr Jordi Vilaró for his help in patient recruitment and evaluation, Dr Rafael Marcos for his valuable statistical advice, Dr Marta Cascante and Dr Vitaly Selivanov for their criticism, Mr Roger Marshall for his editing skills and Sandra Mas for technical assistance.

Funding: The study was supported by BioBridge (LSHG-CT-2006-037939) (EU), Marato TV3 (042010), FIS 03/0062, FIS 05/2486, FIS (06/1510) (Spain) and CibeRes (Instituto de Salud Carlos III, Ministerio de Sanidad) (Spain). EB is a scholar of the Fondo de Investigación Sanitaria (FIS) of the Spanish Health Ministry.

Competing interests: None.

Ethics approval: The Ethics Committee on Human Investigation at Hospital Clinic, Barcelona, Spain, approved all of the experiments.

\section{REFERENCES}

1. Casaburi R, Patessio A, loli $\mathrm{F}$, et al. Reductions in exercise lactic acidosis and ventilation as a result of exercise training in patients with obstructive lung disease. Am Rev Respir Dis 1991;143:9-18.

2. Gosselink R, Troosters T, Decramer M. Exercise training in COPD patients: the basic questions. Eur Respir J 1997;10:2884-91.

3. Maltais F, LeBlanc P, Simard C, et al. Skeletal muscle adaptation to endurance training in patients with chronic obstructive pulmonary disease. Am J Respir Crit Care Med 1996;154:442-7.

4. Sala $\mathbf{E}$, Roca J, Marrades RM, et al. Effects of endurance training on skeletal muscle bioenergetics in chronic obstructive pulmonary disease. Am J Respir Crit Care Med 1999;159:1726-34

5. Rabe KF, Hurd S, Anzueto A, et al. Global strategy for the diagnosis, management, and prevention of chronic obstructive pulmonary disease: GOLD executive summary. Am J Respir Crit Care Med 2007;176: 532-55.

6. Nici L, Donner C, Wouters E, et al. American Thoracic Society/European Respiratory Society statement on pulmonary rehabilitation. Am J Respir Crit Care Med 2006;173:1390-413.

7. Casaburi R, Porszasz J, Burns MR, et al. Physiologic benefits of exercise training in rehabilitation of patients with severe chronic obstructive pulmonary disease. Am J Respir Crit Care Med 1997:155:1541-51.

8. Maltais F, LeBlanc $\mathrm{P}$, Jobin J, et al. Intensity of training and physiologic adaptation in patients with chronic obstructive pulmonary disease. Am J Respir Crit Care Med 1997:155:555-61.

9. Barreiro $\mathbf{E}$, Gea J, Corominas JM, et al. Nitric oxide synthases and protein oxidation in the quadriceps femoris of COPD patients. Am J Respir Cell Mol Biol 2003:29:771-8.

10. Barreiro $\mathbf{E}, \mathrm{Gea}$ J, Matar G, et al. Expression and carbonylation of creatine kinase in the quadriceps femoris muscles of patients with chronic obstructive pulmonary disease. Am J Respir Cell Mol Biol 2005;33:636-42.

11. Barreiro E, de la Puente B, Minguella J, et al. Oxidative stress and respiratory muscle dysfunction in severe chronic obstructive pulmonary disease. Am J Respir Crit Care Med 2005:171:1116-24.

12. Heunks LM, Vina J, van Herwaarden $\mathrm{CL}$, et al. Xanthine oxidase is involved in exercise-induced oxidative stress in chronic obstructive pulmonary disease. Am J Physiol 1999;277:R1697-704.

13. Koechlin C, Couillard A, Simar D, et al. Does oxidative stress alter quadriceps endurance in chronic obstructive pulmonary disease? Am J Respir Crit Care Med 2004;169:1022-7.

14. Rabinovich RA, Ardite E, Troosters T, et al. Reduced muscle redox capacity after endurance training in patients with chronic obstructive pulmonary disease. Am J Respir Crit Care Med 2001;164:1114-18

15. Barreiro E, Galdiz JB, Marinan M, et al. Respiratory loading intensity and diaphragm oxidative stress: N-acetyl-cysteine effects. J Appl Physiol 2006;100:555-63.

16. Rabinovich RA, Ardite E, Mayer AM, et al. Training depletes muscle glutathione in patients with chronic obstructive pulmonary disease and low body mass index. Respiration 2006;73:757-61.
17. Hare JM. Nitroso-redox balance in the cardiovascular system. $\mathrm{N}$ Engl J Med 2004;351:2112-14.

18. Richardson RS, Leek BT, Gavin TP, et al. Reduced mechanical efficiency in chronic obstructive pulmonary disease but normal peak $\mathrm{VO}_{2}$ with small muscle mass exercise. Am J Respir Crit Care Med 2004;169:89-96.

19. Schols AM, Buurman WA, Staal van den Brekel AJ, et al. Evidence for a relation between metabolic derangements and increased levels of inflammatory mediators in a subgroup of patients with chronic obstructive pulmonary disease. Thorax 1996:51:819-24.

20. Barreiro $\mathbf{E}$, Schols $\mathrm{AM}$, Polkey $\mathrm{Ml}$, et al. Cytokine profile in quadriceps muscles of patients with severe COPD. Thorax 2008;63:100-7.

21. Rabinovich RA, Bastos R, Ardite E, et al. Mitochondrial dysfunction in COPD patients with low body mass index. Eur Respir J 2007;29:643-50.

22. Barreiro $\mathbf{E}$, Gea J, Di Falco $\mathrm{M}$, et al. Protein carbonyl formation in the diaphragm. Am J Respir Cell Mol Biol 2005:32:9-17.

23. Coin A, Sergi G, Minicuci N, et al. Fat-free mass and fat mass reference values by dual-energy X-ray absorptiometry (DEXA) in a 20-80-year-old Italian population. Clin Nutr 2008;27:87-94.

24. Gosselink R, Troosters T, Decramer M. Peripheral muscle weakness contributes to exercise limitation in COPD. Am J Respir Crit Care Med 1996;153:976-80.

25. Bernard S, LeBlanc P, Whittom F, et al. Peripheral muscle weakness in patients with chronic obstructive pulmonary disease. Am J Respir Crit Care Med 1998;158:629-34.

26. Barreiro $\mathbf{E}$, Comtois AS, Gea J, et al. Protein tyrosine nitration in the ventilatory muscles: role of nitric oxide synthases. Am J Respir Cell Mol Biol 2002;26:438-46.

27. Montes de Oca M, Torres SH, De Sanctis J, et al. Skeletal muscle inflammation and nitric oxide in patients with COPD. Eur Respir J 2005:26:390-7.

28. Agustí A, Morlá M, Sauleda J, et al. NF-kappaB activation and iNOS upregulation in skeletal muscle of patients with COPD and low body weight. Thorax 2004:59:483-7.

29. Vassilakopoulos T, Deckman G, Kebbewar M, et al. Regulation of nitric oxide production in limb and ventilatory muscles during chronic exercise training. Am J Physiol Lung Cell Mol Physiol 2003;284:L452-7.

30. Souza JM, Daikhin E, Yudkoff M, et al. Factors determining the selectivity of protein tyrosine nitration. Arch Biochem Biophys 1999;371:169-78.

31. Castegna A, Thongboonkerd V, Klein JB, et al. Proteomic identification of nitrated proteins in Alzheimer's disease brain. J Neurochem 2003;85:1394-401.

32. Peluffo G, Radi R. Biochemistry of protein tyrosine nitration in cardiovascular pathology. Cardiovasc Res 2007:75:291-302.

33. Gole MD, Souza JM, Choi I, et al. Plasma proteins modified by tyrosine nitration in acute respiratory distress syndrome. Am J Physiol Lung Cell Mol Physiol 2000:278:L961-7.

34. MacMillan-Crow LA, Crow JP, Kerby JD, et al. Nitration and inactivation of manganese superoxide dismutase in chronic rejection of human renal allografts. Proc Natl Acad Sci U S A 1996:93:11853-8.

35. Castro L, Rodriguez M, Radi R. Aconitase is readily inactivated by peroxynitrite, but not by its precursor, nitric oxide. J Biol Chem 1994;269:29409-15.

36. Souza JM, Choi I, Chen 0 , et al. Proteolytic degradation of tyrosine nitrated proteins Arch Biochem Biophys 2000;380:360-6.

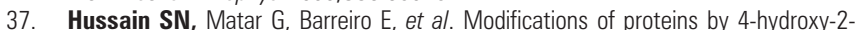
nonenal in the ventilatory muscles of rats. Am J Physiol Lung Cell Mol Physiol 2006;290:L996-1003

38. Fugere NA, Ferrington DA, Thompson LV. Protein nitration with aging in the rat semimembranosus and soleus muscles. J Gerontol A Biol Sci Med Sci 2006;61:806-12.

39. Koeck T, Levison B, Hazen SL, et al. Tyrosine nitration impairs mammalian aldolase A activity. Mol Cell Proteomics 2004;3:548-57.

40. Mihm MJ, Coyle CM, Schanbacher BL, et al. Peroxynitrite induced nitration and inactivation of myofibrillar creatine kinase in experimental heart failure. Cardiovasc Res 2001;49:798-807.

41. Nicolis S, Pennati A, Perani E, et al. Easy oxidation and nitration of human myoglobin by nitrite and hydrogen peroxide. Chemistry 2006;12:749-57.

42. Snook JH, Li J, Helmke BP, et al. Peroxynitrite inhibits myofibrillar protein function in an in vitro assay of motility. Free Radic Biol Med 2008;44:14-23.

43. Dong $\mathbf{M}$, Vongchampa $V$, Gingipalli $L$, et al. Development of enzymatic probes of oxidative and nitrosative DNA damage caused by reactive nitrogen species. Mutat Res 2006;594:120-34

44. Schols AM, Broekhuizen R, Weling-Scheepers CA, et al. Body composition and mortality in chronic obstructive pulmonary disease. Am J Clin Nutr 2005;82:53-9.

45. Vestbo J, Prescott E, Almdal T, et al. Body mass, fat-free body mass, and prognosis in patients with chronic obstructive pulmonary disease from a random population sample: findings from the Copenhagen City Heart Study. Am J Respir Crit Care Med 2006;173:79-83. 